\title{
Natalizumab induces a rapid improvement of disability status and ambulation after failure of previous therapy in relapsing-remitting multiple sclerosis
}

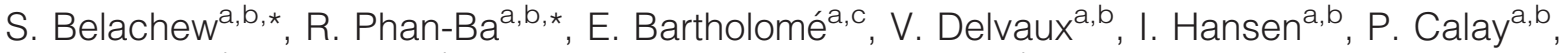 \\ K. E. Hafsi ${ }^{a, d}$, G. Moonen ${ }^{b}$, L. Tshibanda ${ }^{e}$ and M. Vokaer ${ }^{a, d}$ \\ ${ }^{\mathrm{a}}$ MYelin Disorders REseArch teaM (MYDREAM); ${ }^{\mathrm{b}}$ Department of Neurology, C.H.U. Liège University Hospital, Liège; ${ }^{\mathrm{c}}$ Department of \\ Neurology, C.H.U. Tivoli, La Louvière; ${ }^{\mathrm{d} D e p a r t m e n t ~ o f ~ N e u r o l o g y, ~ E r a s m e ~ U n i v e r s i t y ~ H o s p i t a l, ~ B r u s s e l s ; ~ a n d ~}{ }^{\mathrm{e} D e p a r t m e n t ~ o f ~}$ \\ Neuroradiology, C.H.U. Liège University Hospital, Liège, Belgium
}

\section{Keywords:}

alpha-4 integrin, ambulation, disease activity, expanded disability status scale, multiple sclerosis, natalizumab, relapse

Received 4 December 2009 Accepted 7 May 2010
Background: Natalizumab (Tysabri) is a monoclonal antibody that was recently approved for the treatment of relapsing-remitting multiple sclerosis (RRMS). Our primary objective was to analyse the efficacy of natalizumab on disability status and ambulation after switching patients with RRMS from other disease-modifying treatments (DMTs).

Methods: A retrospective, observational study was carried out. All patients $(n=45)$ initiated natalizumab after experiencing at least 1 relapse in the previous year under interferon-beta (IFNB) or glatiramer acetate (GA) treatments. The patients also had at least 1 gadolinium-enhancing $(\mathrm{Gd}+)$ lesion on their baseline brain MRI. Expanded Disability Status Scale (EDSS) scores, and performance on the Timed 25-Foot Walk Test and on the Timed 100-Metre Walk Test were prospectively collected every 4 weeks during 44 weeks of natalizumab treatment. Brain MRI scans were performed after 20 and 44 weeks of treatment.

Results: Sixty-two per cent of patients showed no clinical and no radiological signs of disease activity, and 29\% showed a rapid and confirmed EDSS improvement over 44 weeks of natalizumab therapy. Patients with improvement on the EDSS showed similar levels of baseline EDSS and active T1 lesions, but had a significantly higher number of relapses, and $92 \%$ of them had experienced relapse-mediated sustained EDSS worsening in the previous year. A clinically meaningful improvement in ambulation speed was observed in approximately $30 \%$ of patients.

Conclusions: These results indicate that natalizumab silences disease activity and rapidly improves disability status and walking performance, possibly through delayed relapse recovery in patients with RRMS who had shown a high level of disease activity under other DMTs.

\section{Introduction}

Natalizumab is the first monoclonal antibody approved for the treatment of relapsing-remitting multiple sclerosis (RRMS). The pivotal phase III monotherapy trial in RRMS (AFFIRM) [1] showed that natalizumab can induce a major decrease in the cumulative probability of disability progression. Approximately $37 \%$ of the

Correspondence: S. Belachew, MYelin Disorders REseArch teaM (MYDREAM); Department of Neurology, C.H.U. Liège University Hospital 1 Avenue de l'hôpital, 4000 Liège, Belgium (tel.: + 324 3667255; fax: + 324 3667499; e-mail: sbelachew@ ulg.ac.be).

*These authors contributed equally to this work. treated patients in the AFFIRM trial remained free from combined clinical and radiological disease activity compared with $7 \%$ in the placebo group [2]. Recent observational studies have confirmed a similar range of efficacy of natalizumab treatment [3-8]. Real-life experiences have provided evidence that shifting to natalizumab can improve the control of breakthrough disease insufficiently controlled by interferon-beta (IFNB) and glatiramer acetate (GA) treatments. However, the precise place of natalizumab in the arsenal of MS therapeutics remains debated. The extent to which natalizumab might elicit disability improvement, and in which patients, is also controversial. In Belgium, according to European Medicines Agency (EMEA) 
recommendations [9], natalizumab is indicated in patients with RRMS with an inadequate response to IFNB, or in those initially presenting with a highly active form of relapsing MS.

We report observational data obtained after a period of 44 weeks of natalizumab treatment in a Belgian population composed of patients with radiologically and clinically active MS despite treatment with IFNB or GA. Our primary objective was to analyse classical MS outcomes in our population - annualized relapse rate (ARR), confirmed disability progression assessed by the EDSS, number of new or enlarging T2 lesions, number of $\mathrm{Gd}+\mathrm{T} 1$ lesions and freedom from disease activity. We also planned to perform analyses to identify factors that may determine natalizumab-induced EDSS improvement in a subset of patients [10] and to analyse ambulation performance using the Timed 25-Foot Walk Test (T25FWT) and the Timed 100Metre Walk Test (T100MWT) [11-13].

\section{Methods}

Observational cohort data were collected from two major MS centres in the French speaking part of Belgium (C.H.U. University Hospital of Liège and Erasme Hospital in Brussels). The eligible patient population consisted of men and women, 18-65 years of age, with a diagnosis of RRMS according to the Poser [14] or McDonald [15] criteria. Only patients who had initiated natalizumab at least 44 weeks before the beginning of this study were eligible. Monthly EDSS evaluation was prospectively planned in accordance with the standard procedures of the two centres.

According to the Belgian regulatory provision for natalizumab administration, we included only patients who had experienced at least one relapse in the previous year despite an adequately taken first-line DMT (INFB or GA) and who had at least nine T2 lesions and one $\mathrm{Gd}+\mathrm{T} 1$ lesion on their baseline brain MRI scan. Forty-five patients fulfilled the inclusion criteria. Monthly ambulation assessment was performed in 21 patients from the C.H.U. of Liège.

Treatment consisted of $300 \mathrm{mg}$ of natalizumab by intravenous infusion once every 4 weeks for a period of at least 44 weeks (i.e. 12 doses). Variables analysed included the following: age; gender; disease duration; DMT in the previous year; previous immunosuppressive treatments if applicable; ARR in the previous year and after 44 weeks of natalizumab treatment; history of EDSS changes in the previous year and relapse-induced EDSS worsening with subsequent recovery (complete or incomplete) at the onset of natalizumab therapy; delay between the last relapse and natalizumab onset; EDSS evaluation at baseline and every 4 weeks until the 44th week; number of $\mathrm{Gd}+\mathrm{T} 1$ and new $\mathrm{T} 2$ lesions on brain MRI scans at baseline and after 20 and 44 weeks of natalizumab therapy (brain MRI scans are mandatory every 6 months for natalizumab-treated patients with MS in Belgium); ambulatory performance measured by the T25FWT and T100MWT at each infusion from week $0-44$.

Relapses were defined according to the criteria published by McDonald et al. (2005) [15]: a newly developing symptom or reactivation of a pre-existing deficit for a minimum of $24 \mathrm{~h}$ (in the absence of increased body temperature or infection) occurring at least 30 days after the preceding episode. T2-weighted and T1-weighted (pre- and post-gadolinium infusion) MRI scans of the brain obtained before initiation ( $<3$ months before natalizumab onset) and after 20 and 44 weeks of natalizumab therapy consisted of contiguous, 3-mm-thick axial slices through the whole brain. All adverse events were documented. The study was conducted in compliance with appropriate laws and regulations in Belgium, and no informed consent was needed because of the retrospective nature of the study. As the ambulation tests were part of the standard clinical examination in the C.H.U. of Liège, no informed consent was needed in this respect either.

The T25FWT was performed according to the published instructions of the Multiple Sclerosis Functional Composite score [12,13], and the T100MWT was administered according to previously published guidelines [11]. Note that the T100MWT was performed once, after the T25FWT (2 sessions) and prior to natalizumab infusions.

GraphPad Prism 4 for Macintosh software (Version 4.0b, GraphPad Software Inc., La Jolla, CA, USA) was used to perform statistical analyses (Student's $t$-tests).

\section{Results}

\section{Patients' clinical and radiological baseline characteristics}

Forty-five patients were included in the study (Table 1). There was no drop out during the 44 weeks period of follow-up. The ARR in the previous year, as well as the median disease duration and the EDSS score at baseline appeared to be higher compared to the AFFIRM population (Table 1). Of the 45 patients, 41 (91\%) had experienced at least one EDSS-worsening relapse during the previous year and, of this population, 12/41 (29\%) showed a complete EDSS recovery at the onset of natalizumab treatment. All patients had at least one $\mathrm{Gd}+\mathrm{T} 1$ lesion on their baseline MRI scan compared to only $51 \%$ of the natalizumab-treated cohort in AFFIRM (Table 1). 
Table 1 Patients baseline characteristics

\begin{tabular}{|c|c|c|}
\hline & $\begin{array}{l}\text { MYDREAM } \\
N=45\end{array}$ & $\begin{array}{l}\text { AFFIRM [ref. 1] } \\
\text { Natalizumab } \\
N=627\end{array}$ \\
\hline Age, years (mean $\pm \mathrm{SD}$ ) & $37.0 \pm 10.13$ & $35.6 \pm 8.5$ [ref. 1] \\
\hline Gender, Female (\%) & 73 & 72 \\
\hline $\begin{array}{l}\text { Median disease duration, } \\
\text { years [range] }\end{array}$ & $7.0[1-24]$ & 5.0 [0-34] [ref. 1] \\
\hline $\begin{array}{l}\text { Annual relapse rate in } \\
\text { the previous year } \\
\text { (mean } \pm \mathrm{SD} \text { ) }\end{array}$ & $1.87 \pm 0.73$ & $1.53 \pm 0.91$ [ref. 1] \\
\hline $\begin{array}{l}\text { EDSS worsening relapse } \\
\text { in the previous year } \\
\text { ( } \% \text { of patients) }\end{array}$ & 91 & n.a. \\
\hline $\begin{array}{l}\text { Complete EDSS recovery } \\
\text { from relapses in the previous } \\
\text { year ( } \% \text { of patients) }\end{array}$ & 29 & n.a. \\
\hline EDSS score (mean \pm SD) & $3.5 \pm 1.3$ & $2.3 \pm 1.2$ [ref. 1] \\
\hline $\mathrm{Gd}+$ patients $(\%)$ & 100 & 51 [ref. 1] \\
\hline $\begin{array}{l}\mathrm{Gd}+\mathrm{T} 1 \text { lesions at } \\
\text { baseline }(\text { mean } \pm \mathrm{SD})\end{array}$ & $1.7 \pm 1.2$ & $2.2 \pm 4.7$ [ref. 1] \\
\hline GA in the previous year $(\%)$ & 27 & $3^{\mathrm{a}}$ [ref. 18] \\
\hline IFNB in the previous year $(\%)$ & 73 & $6^{\mathrm{a}}$ [ref. 18] \\
\hline $\begin{array}{l}\text { Previous immunosuppression } \\
\text { (mitoxantrone, azathioprine) }(\%)\end{array}$ & 28 & n.a. \\
\hline
\end{tabular}

SD, standard deviation; ARR, annualized relapse rate; EDSS, Expanded Disability Status Scale; Gd +, gadolinium enhanced; GA, glatiramer acetate; IFNB, interferon beta 1; n.a., not available. ${ }^{a}$ In AFFIRM, the date refer to the previous use of GA and IFNB, not necessarily in the previous year and according to exclusion criteria not in the past 6 months, and not for more than 6 months.

\section{Natalizumab efficacy to control disease activity}

The mean ARR of our population was reduced from 1.87 \pm 0.73 (mean $\pm \mathrm{SD}$ ) at baseline to $0.29 \pm 0.69$ (mean \pm SD) after 44 weeks of natalizumab treatment. The mean EDSS value was $3.5 \pm 1.3$ (mean \pm SD) at baseline and $3.0 \pm 1.3$ (mean $\pm \mathrm{SD}$ ) after 44 weeks of treatment. The mean number of $\mathrm{Gd}+\mathrm{T} 1$ lesions was $1.7 \pm 1.2$ (mean \pm $\mathrm{SD})$ at baseline, and $0.1 \pm 0.3$ (mean \pm SD) and $0.07 \pm$ 0.25 (mean \pm SD) after 20 and 44 weeks of natalizumab therapy, respectively. The mean number of new or enlarged T2 lesions was $0.2 \pm 0.5$ (mean \pm SD) at 20 weeks and $0.22 \pm 0.26$ (mean \pm SD) after 44 weeks of natalizumab therapy.

Freedom from clinical disease activity (i.e. no relapse and no confirmed EDSS worsening at 3 months) was observed in $82 \%(37 / 45)$ of the patients over 44 weeks. Freedom from radiological disease activity (no new or enlarging $\mathrm{T} 2$ lesions and no $\mathrm{Gd}+\mathrm{T} 1$ lesions) applied to $71 \%$ of the patients $(32 / 45)$ combining the results of the 2 MRI scans at 20 and 44 weeks. A composite clinical and radiological freedom from disease activity after 44 weeks of natalizumab treatment was observed in $62 \%(28 / 45)$ of our cohort.

\section{Rapid and sustained EDSS improvement}

During the 44 weeks of follow-up, 18 patients (40\%) remained stable on EDSS at all time points, 12 patients $(27 \%)$ showed a marginal improvement in their EDSS (i.e. 0.5 point improvement or 1.0 point improvement not confirmed at 3 months) and 13 patients (29\%) showed a $\geq 1.0$ point EDSS improvement sustained at 3 months (Fig. 1a and b). Two patients (4\%) experienced a marginal worsening of EDSS ( 0.5 point) under natalizumab therapy, and no patients experienced a 1.0 EDSS worsening sustained at 3 months (Fig. 1b). Nearly all confirmed EDSS changes occurred during the first 20 weeks of treatment (Fig. 1c and d).

\section{Predictive parameters of EDSS improvement}

We performed case-control analyses of EDSS changes under natalizumab therapy based on the ARR in the previous year, the number of $\mathrm{Gd}+\mathrm{T} 1$ lesions on the baseline MRI scan, the baseline EDSS value and EDSS history in the previous year. We also extracted these baseline parameters from patients in whom natalizumab induced a confirmed EDSS improvement compared to patients remaining EDSS stable or experiencing non-sustained EDSS changes.

Of patients with an ARR $\geq 2(n=31), 13(42 \%)$ showed a 3-month-confirmed EDSS improvement under natalizumab therapy, whilst none of the patients with an $\mathrm{ARR}=1(n=14)$ had the same evolution. The mean ARR in the previous year was significantly higher in the EDSS-improving patients $(n=13)$ than in others $(n=32)$, i.e. $2.46 \pm 0.66$ vs. $1.63 \pm 0.61$ (mean $\pm \mathrm{SD}, 95 \%$ confidence interval (CI), 0.43 to 1.3 ; $P<0.001)$, respectively. The proportion of patients experiencing confirmed EDSS improvement was only slightly higher in patients who had two or more $(n=17)$ vs. one $(n=28) \mathrm{Gd}+\mathrm{T} 1$ lesions on the baseline brain MRI scan, (35\% vs. 29\%). Consistently, the mean number of $\mathrm{Gd}+\mathrm{T} 1$ lesion was slightly higher at baseline in the EDSS-improving population, although this was not significant $(1.92 \pm 1.44$ vs. $1.66 \pm 1.1$; mean $\pm \mathrm{SD} ; 95 \% \mathrm{CI},-0.531$ to 1.06 , $P>0.05)$. Regarding the baseline EDSS score, the proportion of patients with confirmed EDSS improvement was $26 \%$ for patients with a mean baseline EDSS $<3.5(n=26)$ and $31 \%$ for patients with an EDSS $\geq 3.5(n=19)$. The mean baseline EDSS value was not significantly different between EDSS-improving patients $(n=13)$ and others $(n=32)(3.65 \pm 1.13$ vs. $3.44 \pm 1.35$; mean $\pm \mathrm{SD} ; 95 \% \mathrm{CI},-0.643$ to 1.08 , $P>0.05)$. Finally, we evaluated the impact of previous EDSS history. Confirmed EDSS improvement under natalizumab therapy was not observed in patients with 

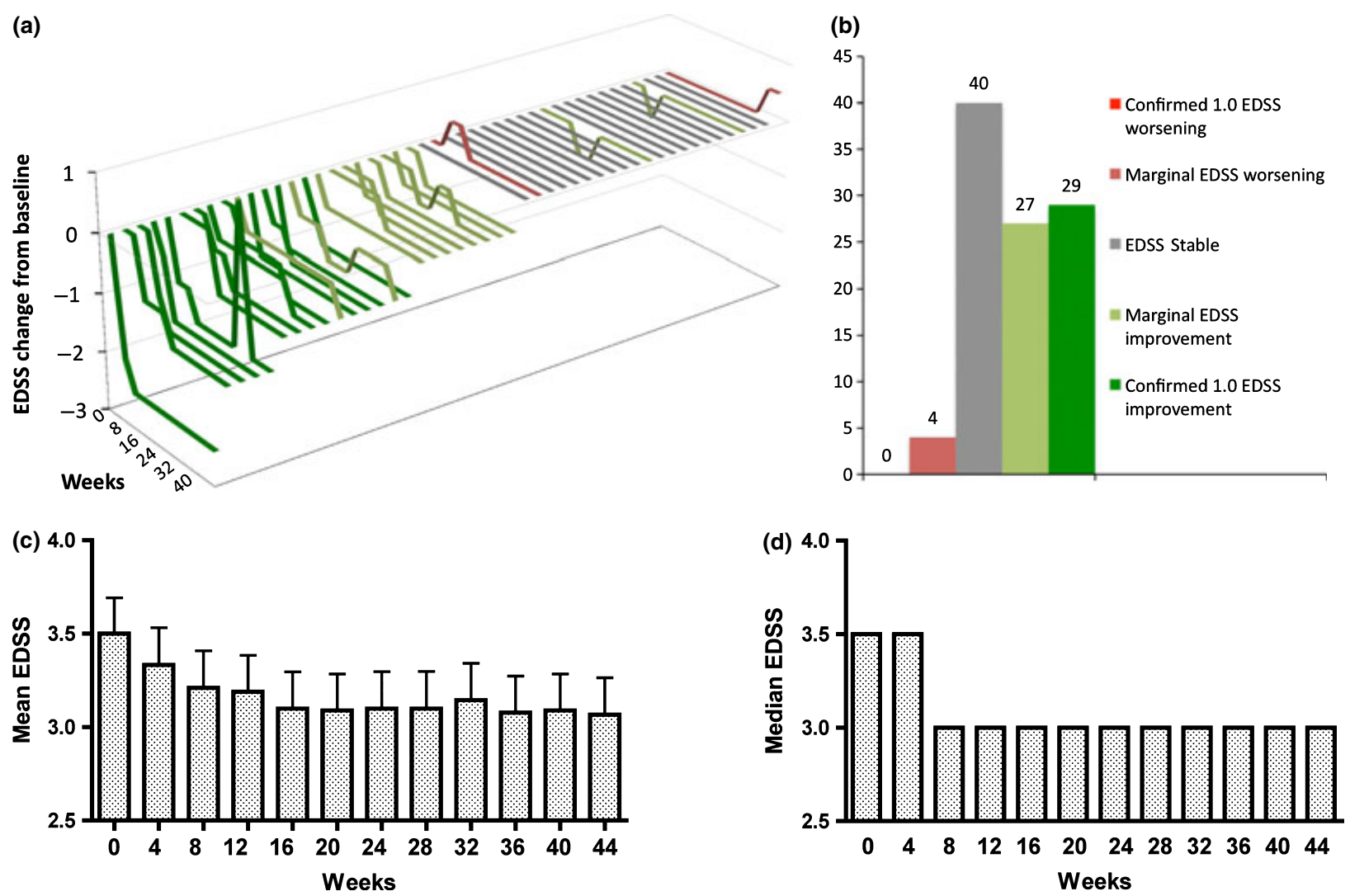

Figure 1 (a) Individual EDSS changes from baseline over 44 weeks of follow-up. Colour codes are consistent with the different subgroups described in panel $b$, with grey lines representing EDSS-stable patients; light green lines for patients experiencing a marginal EDSS improvement; dark green lines for patients experiencing a confirmed EDSS improvement; light red lines for patients experiencing a marginal EDSS worsening; dark red lines for patients experiencing a confirmed EDSS worsening (b) Distribution of EDSS evolution in our population after 44 weeks of natalizumab therapy (c) EDSS evolution (mean \pm SD) over 44 weeks of natalizumab therapy (d) EDSS evolution (median) over 44 weeks of natalizumab therapy.

a stable EDSS in the previous year (no EDSS-increasing relapse in the previous year, $\mathrm{n}=4)$. In contrast, confirmed EDSS improvement was observed in $8 \%$ of patients who had experienced reversible EDSSincreasing relapses in the previous year $(n=12)$, and in $41 \%$ of patients who had sustained EDSS worsening because of relapses in the previous year $(n=29)$. The percentage of patients with a history of sustained EDSS worsening because of relapses in the previous year was $92 \%$ in EDSS-improving patients $(12 / 13)$ and $50 \%$ in others (16/32).

\section{Early and sustained improvement in ambulation}

We performed a thorough analysis of ambulation parameters in a subpopulation of our cohort $(n=21$, patients from the C.H.U. of Liège only, with similar baseline characteristics compared to the global population of 45 patients) (Fig. 2). For the T25FWT (Fig. 2a) and the T100MWT (Fig. 2b), we observed a significant improvement in most patients during the
44 weeks of natalizumab treatment, from a mean value of $5.5 \pm 2.7 \mathrm{~s}$ to $4.3 \pm 1.3 \mathrm{~s}$ (mean $\pm \mathrm{SD} ; 95 \% \mathrm{CI}$, 0.316 to $2.00, P<0.05)$ and $72 \pm 44 \mathrm{~s}$ to $55 \pm 22 \mathrm{~s}$ (mean $\pm \mathrm{SD} ; 95 \% \mathrm{CI}, 3.07$ to $29.1, P<0.05$ ), respectively. Considering a cut-off value of $20 \%$ change as a clinically meaningful change in ambulation performance $[16,17]$, we provided evidence that no patient experienced such a $20 \%$ worsening at 44 weeks of treatment for both tests. In contrast, the proportions of patients who experienced at least $20 \%$ of 3 -monthconfirmed improvement at 20 and 44 weeks of treatment were $33.3 \%$ and $38.1 \%$ for the T25FWT and $28.6 \%$ and $23.8 \%$ for the T100MWT, respectively. All patients who showed a confirmed improvement in their performance on the T100MWT at 44 weeks $(5 / 21)$ also consistently showed a confirmed improvement in T25FWT values $(8 / 21)$. Interestingly, $\pm 50 \%$ of patients who significantly improved their ambulation speed $(4 / 8$ for T25FWT and $2 / 5$ for T100MWT) were completely stable with regard to their EDSS status throughout the 44 weeks of natalizumab treatment. Other patients 
(a)

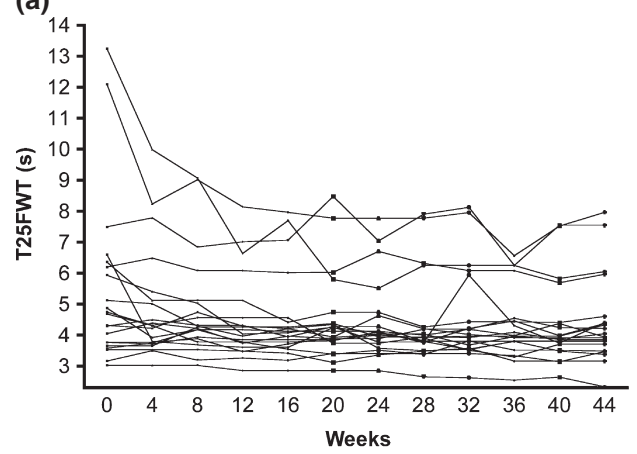

(b)

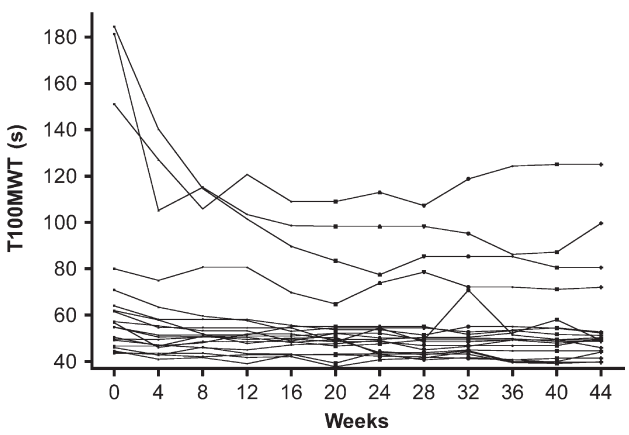

Figure 2. (a) Individual Timed 25-Foot Walk Test (T25FWT) evolution over 44 weeks of natalizumab therapy (b) Individual Timed 100Metre Walk Test (T100MWT) evolution over 44 weeks of natalizumab therapy.

experiencing a confirmed walking improvement $(4 / 8$ for T25FWT and $3 / 5$ for T100MWT) showed a concomitant 3-month-confirmed EDSS improvement at the end of the 44-week observational period.

\section{Adverse events}

No serious adverse events were reported in our cohort during the 44 weeks of natalizumab treatment. Nineteen patients $(42 \%)$ experienced minor adverse events, including upper respiratory tract infections $(n=7)$, fatigue $(n=3)$, infusion reactions $(n=2)$, diarrhoea $(n=3)$, urinary tract infections $(n=1)$, other minor infections $(n=2)$, headache $(n=2)$, spastic angina $(n=1)$, flu-like symptoms $(n=1)$, cutaneous zoster infections $(n=2)$, pruritus $(n=1)$ and muscle cramps $(n=1)$.

\section{Discussion}

The pivotal phase III trials were not designed to examine whether natalizumab can improve disability and walking performance in particular as an escalation therapy. In the present study, the patient population was comparable to the highly active subgroup of the AFFIRM cohort [18] because of local registration criteria. This probably explains why the baseline clinical and radiological features of disease severity were more severe than in the AFFIRM trial.

Our results were consistent with previous findings regarding the established efficacy of natalizumab [3-8]. The clinical, radiological and combined freedom from disease activity appeared to be higher in this study than in previous study populations $[3,6]$, despite a greater stringency in the frequency of clinical and brain MRI assessments.

EDSS improvement has already been described under natalizumab therapy in other cohort studies $[3,6]$ and post hoc analyses from the AFFIRM trial [10]. We observed here that $29 \%$ of patients showed a natalizumab-mediated confirmed EDSS improvement during the 11 months of observational follow-up. We performed an analysis of EDSS improvement related to EDSS history. This revealed that $41 \%$ of patients who had experienced a sustained EDSS worsening because of relapses in the previous year showed a confirmed EDSS improvement under natalizumab therapy, in contrast to only $8 \%$ of the population having experienced a transient EDSS worsening and $0 \%$ of the population with a stable prior EDSS course. Hence, EDSS history in the previous year seems to be a critical factor in determining the fate of EDSS evolution throughout natalizumab therapy. On the other hand, this study did not reveal MRI parameters that may predict clinical improvement under natalizumab therapy.

Beyond the EDSS, we also studied the ambulation capacities of our population, because walking is obviously a crucial component of the quality of life of patients with MS. It is noteworthy that EDSS does not take ambulation into account below a score of 4.0. We used instead a longer distance walking test, the Timed 100-Metre Walk Test (T100MWT), which in addition to reflecting speed, may be more sensitive to essential regulators of the actual maximal walking distance such as gait and spasticity-related fatigue. After 11 months of follow-up, $38 \%$ of patients had improved by more than $20 \%$ on the T25FWT and $23 \%$ had improved by more than $20 \%$ on the T100MWT. It is important to note that half of the patients who significantly improved their ambulation speed in both tests remained EDSS stable throughout the 44 weeks of clinical assessment.

EDSS and ambulation improvement under natalizumab therapy may be attributed to reduced inflammation and resolution of oedema besides putative tissue repair and plasticity to a limited extent. This improvement seems to be critically linked to the history of EDSS during the previous year, and further prospective 
larger-scale and longer-term trials are needed to elucidate its precise mechanisms.

\section{Acknowledgement}

P. Calay was supported by an educational grant from Biogen Idec (Belgium).

\section{References}

1. Polman CH, O'Connor PW, Havrdova E, et al. A randomized, placebo-controlled trial of natalizumab for relapsing multiple sclerosis. $N$ Engl J Med 2006; 354: 899 910.

2. Havrdova E, Galetta S, Hutchinson M, et al. Effect of natalizumab on clinical and radiological disease activity in multiple sclerosis: a retrospective analysis of the Natalizumab Safety and Efficacy in Relapsing-Remitting Multiple Sclerosis (AFFIRM) study. Lancet Neurol 2009; 8: 254-260.

3. Putzki N, Yaldizli O, Maurer M, et al. Efficacy of natalizumab in second line therapy of relapsing-remitting multiple sclerosis: results from a multi-center study in German speaking countries. Eur J Neurol 2010; 17: 31-37.

4. Putzki N, Kollia K, Woods S, Igwe E, Diener HC, Limmroth V. Natalizumab is effective as second line therapy in the treatment of relapsing remitting multiple sclerosis. Eur J Neurol 2009; 16: 424-426.

5. Putzki N, Yaldizli O, Buhler R, Schwegler G, Curtius D, Tettenborn B. Natalizumab reduces clinical and MRI activity in multiple sclerosis patients with high disease activity: results from a multicenter study in Switzerland. Eur Neurol 2010; 63: 101-106.

6. Outteryck O, Ongagna JC, Zephir H, et al. Demographic and clinic characteristics of French patients treated with natalizumab in clinical practice. J Neurol 2010; 257: 207211.

7. Tedeschi G, Amato MP, D'Alessandro R, et al. The pharmacovigilance program on natalizumab in Italy: 2 years of experience. Neurol Sci 2009; 30(Suppl 2): S163S165.
8. Oturai AB, Koch-Henriksen N, Petersen T, Jensen PE, Sellebjerg F, Sorensen PS. Efficacy of natalizumab in multiple sclerosis patients with high disease activity: a Danish nationwide study. Eur J Neurol 2009; 16: 420-423.

9. European Medicines Agency (2006). http://www. emea.europa.eu/humandocs/PDFs/EPAR/tysabri/H-603en6.pdf (accessed November 2009).

10. Munschauer F, Giovannoni G, Lublin F, et al. Natalizumab significantly increases the cumulative probability of sustained improvement in physical disability. ACTRIMS/ECTRIMS/LACTRIMS; 17-20/9/2008; Montreal, 2008.

11. Belachew S, Calay P, Delvaux V, et al. The timed 100-meter walk test: an easy to use sensitive tool to detect and evaluate restricted walking capacities in multiple sclerosis. ACTRIMS/ECTRIMS/LACTRIMS; 17-20/9/2008; Montreal, 2008.

12. Cutter GR, Baier ML, Rudick RA, et al. Development of a multiple sclerosis functional composite as a clinical trial outcome measure. Brain 1999; 122(Pt 5): 871-882.

13. Fischer JS, Rudick RA, Cutter GR, Reingold SC. The Multiple Sclerosis Functional Composite Measure (MSFC): an integrated approach to MS clinical outcome assessment. National MS Society Clinical Outcomes Assessment Task Force. Mult Scler 1999; 5: 244-250.

14. Poser CM, Paty DW, Scheinberg L, et al. New diagnostic criteria for multiple sclerosis: guidelines for research protocols. Ann Neurol 1983; 13: 227-231.

15. Polman $\mathrm{CH}$, Reingold SC, Edan G, et al. Diagnostic criteria for multiple sclerosis: 2005 revisions to the "McDonald Criteria". Ann Neurol 2005; 58: 840-846.

16. Bosma LV, Kragt JJ, Brieva L, et al. The search for responsive clinical endpoints in primary progressive multiple sclerosis. Mult Scler 2009; 15: 715-720.

17. Kragt JJ, van der Linden FA, Nielsen JM, Uitdehaag BM, Polman CH. Clinical impact of $20 \%$ worsening on Timed 25-foot Walk and 9-hole Peg Test in multiple sclerosis. Mult Scler 2006; 12: 594-598.

18. Hutchinson M, Kappos L, Calabresi PA, et al. The efficacy of natalizumab in patients with relapsing multiple sclerosis: subgroup analyses of AFFIRM and SENTINEL. J Neurol 2009; 256: 405-415. 